\title{
Opinions on the ASCO 2011 Annual Meeting
}

\author{
Chair: Rupert Bartsch ${ }^{\mathrm{a}}$ \\ Participants: Peter C. Dubsky ${ }^{b}$ Sibylle Loibl ${ }^{c}$ Günther Steger ${ }^{a}$ \\ aKlinische Abteilung für Onkologie, Universitätsklinik für Innere Medizin I, Medizinische Universität Wien, \\ bUniversitätsklinik für Chirurgie, Medizinische Universität Wien, Austria \\ ${ }^{\circ} \mathrm{GBG}$ Forschungs GmbH, Neu-Isenburg, Germany
}

\section{Question 1: In Your Opinion, What Was the Most Relevant New Finding in the Field of Breast Cancer Presented at the 2011 ASCO Annual Meeting?}

Dubsky: I was very impressed with the NCIC CTG MAP.3 study. Exemestane was able to reduce the annual risk of breast cancer by $65 \%$ in a cohort of postmenopausal women with limited risk. The toxicity data are very favorable; even though the quality of life differences are rather minute. It is noteworthy that e.g. age above 60 was enough of a risk factor to be included in this trial. The median Gail risk score was $2.3 \%$ - despite these criteria the study comfortably reached its endpoint. I think it is with this background in mind that we should consider the data: Clearly, treating 2,285 patients for around 3 years with a fairly expensive drug to avoid 21 invasive breast cancers is not something that health authorities will look upon with great kindness. However, we should keep in mind that the prevention effect is likely to be much higher in e.g. postmenopausal patients with remaining high breast density and clear family history of breast cancer. To these patients, who on top of being objectively at risk often display strong cancer anxiety, we should be offering this drug. Furthermore, we have learned from other aromatase inhibitors that the expense of a drug is also a matter of time indeed, exemestane will be a very affordable treatment as patents end.

The MA.20 trial addressed the long-standing issue of adding regional nodal irradiation (RNI) to whole breast irradiation (WBI) in patients with positive lymph nodes or high-risk node negative disease. Not surprisingly the investigators found a reduced incidence of local recurrences in patients with 1-3 positive lymph nodes. However, I was struck by the clear advantage in disease free survival (DFS) and the decreased rate of distant metastases. It is very likely that overall survival (OS) was improved for some of these women. There are several issues that urgently need to be addressed: These data are clearly in contrast to the EBCTCG overview for several reasons: There is a $4.3 \%$ improvement in distant metastases whereas the local recurrence rate (LRR) benefit was $2.3 \%$. This is against the previous paradigm that at least $10 \%$ decrease of LRR needs to be achieved to have a significant shift in OS. Furthermore, the distant metastasis survival curves separate early. In fact, they seem to separate in parallel to the LRR curves. This trial should be considered practice changing. RNI should be considered for patients with higherrisk tumors and 1-3 positive lymph nodes.

Among the many interesting reports during the ASCO 2011 I would simply like to point out two other subject areas: 1) Dual inhibition of the Her-2 receptor is a very effective neoadjuvant treatment. It is also striking how effective these treatments are in the absence of cytototoxic treatment. Future research should be directed in defining a subgroup of patients that can be treated without the addition of chemotherapy. This is an exciting direction of clinical research. 2) The German Breast Cancer Group did not receive much attention for a highly interesting abstract concerning the post-neoadjuvant survival of patients (abstract 1028). These data include the survival of over 6,300 women with a median follow-up of 42 months and should be studied with great care, since they are likely to influence many of our treatment and follow-up choices after patients have undergone preoperative chemotherapy.

Loibl: The data from the MAP.3 study investigating exemestane for the prevention of breast cancer in women with an increased risk. The number needed to treat to prevent one case of invasive breast cancer was extremely positive $(n=26$ in 5 years and 94 in 3 years). This is practice changing. The

\section{KARGER}

Fax +497614520714

Information@Karger.de

www.karger.com
(C) 2011 S. Karger GmbH, Freiburg

$1661-3791 / 11 / 0064-0315 \$ 38.00 / 0$

Accessible online at:

www.karger.com/brc
PD Dr. med. Rupert Bartsch

Universitätsklinik für Innere Medizin I / Comprehensive Cancer Center

Klinische Abteilung für Onkologie, Medizinische Universität Wien

Währinger Gürtel 18-20, 1090 Wien, Austria

Tel. +43 1 40400-4445, Fax -4451

rupert.bartsch@meduniwien.ac.at 
data generated by this clear and clean trial are amazing. The data are already available with free access in the New England Journal of Medicine and are accompanied by an Editorial from Nancy Davidson and Thomas Kensler ending with the words '... breast cancer is the second most common cause of death from cancer and one of the most feared diagnoses for women in the United States. We have the knowledge and tools to reduce its incidence today. We have run out of excuses. What are we waiting for?' Data from the IBIS II trial which is still recruiting patients are needed in order to confirm the results and start a new era of chemoprevention in menopausal women.

Steger: As expected, no major break-through advances for breast cancer treatment or diagnosis were presented. However, the prevention data with exemestane presented by Paul Goss add to our possibilities in an important area of the field.

\section{Question 2: New Results of PARP Inhibition as Treatment for Triple Negative Breast Cancer Were Somewhat Disappointing. What in Your Opinion Is Currently the Most Promising Approach for Targeting Triple Negative and Basal-Like Tumors? Is There a Future Role for PARP Inhibition?}

Dubsky: Joyce O'Shaugnessy presented the data of a randomized phase III study of iniparib (BSI-201) in combination with gemcitabine/carboplatin $(\mathrm{G} / \mathrm{C})$ in metastatic triple negative breast cancer (TNBC; Abstract: 1003). Just how 'disappointed' do we have a right to be? I am not convinced that what has been presented is really qualitatively distinct from the prominently published phase II study with BSI-201. The population and the treatment are very similar, but all the requirements of a phase III trial plus an ambitious statistical design apply. The clinical activity of iniparib was shown to be less than predicted - does this mean the drug is inactive? First, defining a subgroup via the absence of markers is a poor selection criterion. The trial has announced several tissue based translational protocols - including genotyping. This will be informative for future trial designs. Furthermore, the proposed chemotherapy backbone of $\mathrm{G} / \mathrm{C}$ is an unusual regimen in our local practice. Intertrial comparisons suggest that this may not be the best possible treatment. PARP inhibition currently has no role in the metastatic setting of breast cancer outside of clinical trials. In our setting, conventional agents (and not a $\mathrm{G} / \mathrm{C}$ regimen) will remain the main pillar of treatment in TNBC.

Loibl: I believe that there will be a future role for PARP inhibitors. But we have to carefully select the patients and the treatment options. The population included into the phase III registration trial for iniparib had a very bad performance status ( $5 \%$ deaths on study). If we are not carefully selecting the patients for the treatment we might kill a drug before it can really show its potential. Is $\mathrm{G} / \mathrm{C}$ really the best chemotherapy to be used in breast cancer patients? Few people really consider this combination an option in breast cancer treatment. I think we have to go back to square one with this drug. Other compounds, such as olaparib have shown promising data in patients with BRCA mutated tumors. However, the toxicity profile indicates that there will be difficulties in combination with chemotherapy. More data are needed before we can a make a decision on PARP inhbitors. Let's to go back to the drawing board.

Steger: The PARP inhibitor story and the recently published results should remind us again that premature euphoria is never a good guidance in clinical medicine and in oncology in particular. I think that the books are not closed for the PARP inhibitors, but for the time being it appears that for triple negative tumors taxan and anthracyclin based regimens are still the standard of care outside clinical trials.

\section{Question 3: What in Your Opinion Is the Current Role of VEGF Inhibition in Early and Advanced Stage Breast Cancer?}

Dubsky: The role of VEGF inhibition or more specifically of bevacizumab (BEV) is currently being explored in 3 large randomized trials that will cover Her-2 positive, Her-2 negative and triple negative cancer, respectively. There is currently no role of BEV or any of the primarily antiangiogenic compounds in the adjuvant setting.

In advanced breast cancer I experience a contrast between daily practice and clinical research: In the clinic I did see patients with aggressive metastatic disease who showed longterm response to taxane based chemotherapy in combination with BEV. However, clinical trials in this setting have repetitively produced significant increases in progression free survival (PFS) but have failed to show an increase in OS; despite several attempts to show this effect in metaanalyses.

The exploratory analysis of the TNBC subpopulation in RIBBON-2 presented by A. Brufsky (abstract 1010) is yet another successful attempt at showing what could well be a higher benefit of BEV in this subset. The sum of results of TNBC subsets treated with BEV in E2100, AVADO, RIBBON-1 and perhaps ATHENA will certainly increase the willingness of physicians to add BEV in this aggressive type of disease. Although clearly none of these trials have formally shown significant interaction between TNBC and antiangiogenic treatment. In our local practice, we would consider patients with indication to first line taxane-based chemotherapy without clear indication for combination chemotherapy as candidates for paclitaxel with addition of BEV. 
Loibl: There is definitely a place. The addition of BEV to paclitaxel as first line therapy is a licensed option and the data in the metastatic setting are consistent. BEV is currently given to all HER2- patients. But we do not know yet who will really benefit. There is no extra benefit for the triple negative group. However, the neoadjuvant data from the German Breast Group suggest a benefit only for the triple negative patients and the NSABP-B40 study demonstrated an overall benefit which is driven by the hormone receptor positive patients. The data on sorafenib demonstrated that PFS was better with sorafenib in combination with chemotherapy than with chemotherapy alone. The risk reduction was $45 \%$. But the PFS without sorafenib was only 2.7 months. The added toxicity is high. The currently running phase III trial did reduce sorafenib to $600 \mathrm{mg} / \mathrm{d}$. We investigated the combination of chemotherapy (epirubicin, cyclophosphamide, paclitaxel) and sorafenib in the neaoadjuvant setting and found that an individual dose escalation based on the patient's individual toxicity is probably the preferred option. We need to do more translational research and to find the right patient population.

Steger: For early disease I currently do not see a role of VEGF inhibition in daily practice. Based on clinical trial results such as E2100, RIBBON-2, and the now presented data by Ito and colleagues at ASCO, bevacizumab has clinical relevance in advanced disease.

\section{Question 4: Is Axillary Lymph Node Dissection Still the Standard of Care in Patients with Positive Sentinel Lymph Nodes?}

Dubsky: The question should be answered from two primary directions: 1) What is the tumor load in the 'positive' sentinel? There is fairly good evidence to show that isolated tumor cells and micrometastatic disease in sentinel lymph nodes may not be relevant for local or distant recurrences if patients receive adjuvant systemic treatment. At our institution we have stopped performing axillary dissection (AD) in these patients. Indeed, the St. Gallen panel of 2011 clearly favored this approach. 2) If 1 or 2 sentinel lymph nodes show macrometastatic disease, what is the biology of the tumor? Since ASCO 2010 Dr. Giuliano and colleagues have elegantly shown that in a subgroup of patients with favorable tumor profiles $(69 \% \mathrm{~T} 1,80 \% \mathrm{ER}+$ and $\mathrm{cN} 0)$ that have received whole breast irradiation (WBI) and systemic adjuvant therapy, $\mathrm{AD}$ is more likely to be associated with morbidity than with improved regional outcome or patient survival. The authors have always clearly stated that these data may not apply to patients with higher tumor loads, aggressive biology or missing WBI. Indeed, at ASCO 2011 Dr. Whelan presented the MA.20 trial (as discussed above). Considering the many favorable factors associated with the node positive ACOSOG cohort and comparing these risk factors with the MA.20 population it is exemplary of just how different patient risk and response to a local treatment can be despite similar nodal status. Baseline characteristics of MA.20 show that around half of the patients had tumors larger than $2 \mathrm{~cm}$, approximately $40 \%$ were G3, 25.5\% were ER negative, and over $90 \%$ received adjuvant chemotherapy. In this trial axillary dissection (mean lymph node number 12!) with WBI+RNI delivered impressive results concerning DFS and distant metastases. In summary, although macrometastatic involvement of 1 or 2 sentinel nodes should not automatically lead to AD, the omission of axillary surgery should be restricted to patients with a low risk of local failure and favorable tumor biology.

Loibl: I am not sure. The data say we don't need to operate. But these patients will all be irradiated because the radiooncologic guidelines call for irradiation in case of incomplete axillary dissection in patients with axillary lymph node involvement. In the German guidelines we have included a statement that there is no need for complete axillary dissection in those patients who fulfill the entry criteria of the study presented at the SABCS 2011. All women will benefit from radiotherapy of the loco-regional nodes. A simple message brought to us by the radio oncologist Dr. Whelan. The data from the MA.20 study need to be taken into account. Surgery and radiotherapy need to be combined to find the best option for the patients. Interestingly mainly distant relapses could be prevented by irradiating the loco-regional nodes. Therefore the question is not does the patient need an axillary lymph node dissection but how to treat the locoregional nodes in order to prevent distant and local relapses.

Steger: Yes, in my opinion the data are not so unequivocally clear that we can routinely dismiss axillary dissection if a sentinel node is positive.

\section{Question 5: What Is the Optimal Endocrine Treatment Approach in Premenopausal Patients with Early Stage Breast Cancer?}

Dubsky: In Austria we have adhered to the combination of ovarian suppression with LHRH analogs in combination with tamoxifen ever since this regimen was introduced to the clinical trial landscape (starting with ABCSG 5). Currently, most physicians use this combination of goserelin (Zoladex) and tamoxifen for a duration of 3 years. Until data from the SOFT and TEXT trials conducted by the ABCSG are available, very little is likely to influence the ongoing discussion between tamoxifen monotherapy for 5 years vs. differing lengths of the combination regimen. After the negative report from the AZURE trial, the ABCSG reported an update of Trial 12 previously published in 2009. In this 
update, the initial data have matured and reaffirmed both the increased DFS in the patient group treated with zoledronic acid in addition to endocrine therapy and confirmed the non-significant trend in OS. Interestingly, the subgroup of patients older than 40 years derived a higher benefit than younger patients. The anticancer benefits of adjuvant zoledronic acid may be restricted to a group of patients with a low estrogen environment as has previously been shown in the AZURE trial.

Loibl: Have I missed something? I would still say tamoxifen or the combination of an LHRH analogue with tamoxifen.

Steger: ABCSG-12 has now shown a significant survival benefit. Thus, ovarian ablation plus tamoxifen is a valid standard of care in this indication if no clear indication for adjuvant chemotherapy and/or immunotherapy, such as HER2-positivity, is also present.

\section{Question 6: What Role do Multigenomic Assays Currently Have for the Assessment of Recurrence Risk and Prediction of Treatment Response?}

Dubsky: Multigenomic assays are robust tests that indicate prognosis of patients and clearly add to the information that classic pathology currently provides. These assays help to define a large subgroup of ER+ patients with very low risk. These patients are unlikely to benefit from treatments (in most cases chemotherapy) other than surgery, radiotherapy and endocrine treatment. This identification of a lowrisk group of patients is an important milestone in breast cancer treatment and should be used when classification is difficult or unclear after classic pathologic assessment of the specimen.

Currently data that provide prediction of treatment response - more specifically chemotherapy response - are lacking. This is true especially in our ER+ patients. Although both the Mamaprint score and the RS score clearly indicate that $\mathrm{ER}+$ patients at higher risk derive more benefit from chemotherapeutic treatment, it is currently unclear if these multigenomic assays have a better performance in predicting chemotherapy response than a simple algorithm of e.g. ER, grading and KI67. In our multidisciplinary setting we are currently implementing the use of multigenomic assays in a defined subgroup of ER+ patients where we believe chemotherapy treatment decisions can be complemented.

Loibl: This is a very good question. I think multigenomic assays are good for assessing the risk of recurrence, especially in ER+ cohorts. But the predictive ability for therapy indication is not yet validated enough. Is the recurrence score more informative than Ki67?

Steger: Currently we only use them in some patients with hormone-sensitive disease to 'prove' to the patients that no rationale for additional chemotherapy exists. However, in most cases this judgment can be reached by interpretation of the hormone-receptors, Ki67, and grade. I would prefer to have the results of the MINDACT and TaylorX studies before we assign a definitive role to these expensive assays.

\section{Question 7: In Your Opinion, What Is the Most Pertinent Endpoint in Phase II and III Clinical Trials in Advanced Stage Breast Cancer?}

Dubsky: A phase II trial can ask an array of questions that may range from the proof of principle concerning the mechanism of action of a drug to simply wanting to generate enough data to move a new drug into larger randomized trials. Depending on the question the pertinence of an endpoint will change. However, no matter what endpoint we use it is vital that we agree upon definitions of that endpoint. In the adjuvant setting the 'STEEP' system as introduced by C. Hudis and colleagues has been a great help in defining the events that actually contribute to survival endpoints such as DFS, relapse free survival etc. Currently, a similar effort is made by a working group of members from both the Breast International Group (BIG) and the North American Breast Cancer Groups (NABCG). This endeavor will contribute to a better understanding of trial results and give a better grasp on just what type of events constitute 'progression'. In phase III trials OS is certainly not the only pertinent endpoint. But clearly it is the goal we should be setting ourselves to improve care in the advanced treatment setting.

Loibl: In earlier phases PFS because due to multiple therapeutic interaction and cross-over the single effect is difficult to assess with OS unless the treatment effect is huge. In later lines OS is probably the better endpoint.

Steger: Finally, this important discussion, not only but also driven by the recently published trial results with PARP inhibitors and bevacizumab, has begun. Before these discussions will come to hopefully relevant results, I think that for phase II studies the reduction in the hazard for progression by $30 \%$ and a hazard ratio of 0.8 for OS in phase III trials with a mandatory quality of life evaluation in parallel are clinically relevant endpoints. 


\section{Participants}

PD Dr. med. Peter C. Dubsky

Universitätsklinik für Chirurgie

Medizinische Universität Wien

Währinger Gürtel 18-20, 1090 Wien, Austria

Tel. +431 40400-6916, Fax -6918

peter.dubsky@meduniwien.ac.at

PD Dr. med. Sibylle Loibl

GBG Forschungs GmbH

Martin-Behaim-Str. 12, 63263 Neu-Isenburg, Germany

Tel. +49 6102 7480-0, Fax -440

Sibylle.Loibl@germanbreastgroup.de

Univ. Prof. Dr. Günther Steger

Klinische Abteilung für Onkologie

Universitätsklinik für Innere Medizin I

Medizinische Universität Wien, 1090 Wien, Austria

Tel: +43 1 40400-4445, Fax -6081

guenther.steger@meduniwien.ac.at 